\title{
International standards for risk management in nanotechnology
}

To the Editor - It was gratifying to see the article 'The US must help set international standards for nanotechnology' by Vladimir Murashov and John Howard, highlighting the role of voluntary, consensus-based international standards for the risk management of nanotechnology ${ }^{1}$. As chairman of the technical committee for nanotechnologies set up by the International Organisation for Standardization (ISO), I can confirm that the US is already very actively involved in the work of this committee (ISO TC 229) and its four working groups. At present, the committee has about 30 active projects and has a leading role in efforts to coordinate and harmonize work in this area among other international and regional standardization committees, and other organizations with an interest in nanotechnology.

However, the purpose of this response is not to highlight the important work being undertaken by the committee, but to make clear that the suggestion by Murashov and Howard - that the UK could successfully "convert" a national, voluntary standard (British Standards Institution (BSI) Published Document PD 6699-2) into an international standard against the wishes of 32 other countries who are voting members of the committee - shows a serious misunderstanding of the rigorous processes enshrined in the ISO/IEC Directives ${ }^{2}$, to which all 160 members of ISO subscribe. As chairman of the committee that developed this document, I want to make two points.

First, the document has the status of a BSI Published Document, not a full British Standard, and the foreword to the document clearly states that "as a guide, this Published Document takes the form of guidance and recommendations. It should not be quoted as if it were a specification and particular care should be taken to ensure that claims of compliance are not misleading."

Second, in its scope the document "recognizes that there is considerable uncertainty about many aspects of effective risk assessment of nanomaterials, including the hazardous potential of many types of nanoparticles and the levels below which individuals might be exposed with minimal probability of adverse health effects. The guide therefore recommends a cautious strategy for handling and disposing of nanomaterials."

This approach is entirely consistent with that promoted by almost all international organisations, including the National Institute for Occupational Safety and Health (NIOSH) in the US. The guide makes it clear that existing risk management protocols rely on having "good information about the hazardous nature of materials, the effectiveness of control approaches and convenient and accessible ways to monitor exposure", and that "given the lack of current knowledge about the toxicity of nanomaterials and the concern that current safety data sheets do not adequately reflect the hazardous nature of nanomaterials, it is recommended that all nanomaterials are considered potentially hazardous unless sufficient information to the contrary is obtained."

Regarding the proposed benchmark exposure levels in section 8.3 of the document, it clearly states that "the following benchmark exposure levels have been suggested for the four nanoparticle hazard types identified in 7.1. These are intended to provide reasonably cautious levels and are based in each case on the assumption that the hazard potential of the nanoparticle form is greater than the large particle form. This assumption will not be valid in all cases. Although these benchmark levels relate to current exposure limits, they have not been rigorously developed. Rather, they are intended as pragmatic guidance levels only and should not be assumed to be safe workplace exposure limits".

The document was developed to provide timely and pertinent advice to industry and other relevant organisations on current thinking about the precautions that should - in the absence of protocols and regulations relevant to health and environmental toxicology of nanoparticles - be adopted when handling and disposing of engineered nanoparticles. As with all other standards documents, it is subject to regular review and updating to take account of the current state-of-the-art. This document and eight related documents are freely available on the BSI website ${ }^{3}$.

The purpose of using the guide as the basis of a new work item proposal to the ISO technical committee for nanotechnologies was not, as suggested by Murashov and Howard, to gain an ISO "stamp of approval" for the recommendations it contains. The purpose was to encourage members of ISO to develop a document giving clear, practical guidance on "the safe handling and disposal of manufactured nanomaterials" backed by the core principle of international consensus that is the foundation of all the standards published by the three international standards organisations ISO, the International Electrotechnical Commission and the International Telecommunications Union.

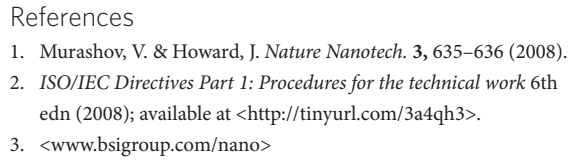

\section{Peter Hatto}

IonBond Limited, Consett, Durham DH8 6TS, UK e-mail: peter.hatto@ionbond.com
Murashov and Howard reply - We thank Peter Hatto for reminding readers about the principle of consensus on which the International Organisation for Standardization (ISO) process is based. In fact, the main objective of our article ${ }^{1}$ was to ensure that such a consensus is based on informed decisions and is reached as a result of all stakeholders and experts in the global community participating actively in the ISO process.

We would like to reiterate that in the presence of incomplete risk information about nanomaterials, prudent measures should be taken to minimize exposure in the workplace. However, this does not require occupational exposure limits (OELs) to be established before any actions can be taken and, given the limited amount of health information, it may be premature to recommend OELs for specific nanomaterials. The problem with "assumed" OELs - such as those described in BSI PD 6699-2 - is that they 